\title{
The results of rapid source control laparotomy or open abdomen for acute diverticulitis
}

\author{
Arthur Berg ${ }^{1} \cdot$ Matthew Rosenzweig ${ }^{1} \cdot$ Yen-Hong Kuo ${ }^{1} \cdot$ Ayolola Onayemi $^{1} \cdot$ Shawla Mohidul ${ }^{2} \cdot$ Micaela Moen $^{2}$. \\ Jason Sciarretta ${ }^{3} \cdot$ John Mihran Davis ${ }^{4,5} \cdot$ Nasim Ahmed $^{1}$
}

Received: 8 January 2021 / Accepted: 16 August 2021 / Published online: 28 August 2021

(c) The Author(s), under exclusive licence to Springer-Verlag GmbH Germany, part of Springer Nature 2021

\begin{abstract}
Introduction Rapid source control laparotomy (RSCL) for the management of non-traumatic intra-abdominal emergencies has increased over the past 25 years when it was advocated for trauma patients. Little data, however, support its widespread use. We hypothesize that the patients with RSCL will have poorer outcomes than those treated with primary fascial closure (PFC).

Methods Patients operated for acute diverticulitis from 2014 to 2016 using The American College of Surgeons sponsored National Surgical Quality Improvement Program (NSQIP) data were reviewed. Two groups were identified: PFC, patients with their closed fascia but skin left open (PFC) and RSCL, patients with their left open fascia after the initial operation. The primary outcome of the study was 30-day mortality, with secondary analyses evaluating complications, discharge location and length of stay. Univariate analysis was initially performed followed by propensity score matching.

Results A total of 460 patients were surgically treated for Hinchey IV diverticulitis of whom 101 (21.9\%) had RSCL. The length of stay of the RSCL patients was significantly longer (15 versus 12 days, $p, 0.02)$ than patients in the PFC group. Similarly, the discharge destination for the PFC group was twice as likely to be discharged home as the RSCL group.

Conclusion RSCL for acute diverticulitis is a widely used but is associated with prolonged hospitalizations resulting in high rates of discharge to skilled nursing or rehabilitation facilities. Its routine use for diverticulitis should be limited.
\end{abstract}

Keywords Damage control laparotomy $\cdot$ Peritonitis $\cdot$ Perforated diverticulitis

\section{Introduction}

Diverticulitis affects a significant percent of the population in Western Europe and North America. It is estimated that 10 to $20 \%$ of the population over the age of 60 years will manifest symptoms and a significant number will require

John Mihran Davis

jmihrandavis@gmail.com

1 Hackensack Meridian Health, Jersey Shore University Medical Center, Neptune, NJ, USA

2 Grand Strand Medical Center, Myrtle Beach, SC, USA

3 Emory School of Medicine, Grady Memorial Hospital, Atlanta, GA, USA

4 South Shore University Hospital - Northwell Health, Bay Shore, NY, USA

5 South Shore University Hospital - Northwell Health, 301 East Main Street, NY 17061 Bay Shore, USA surgery [1-3]. The current incidence of operative diverticulitis is approximately 33,500 patients per year and has increased in the recent past $[2,3]$. This surgery carries with it a very high morbidity rate of 30 to $50 \%$ and a high mortality rate of 10 to $20 \%$ [4]. The standard therapeutic approach has been a staged procedure with resection of the diseased segment with an end colostomy and stapled distal segment, the Hartmann's procedure [3]. More recent approaches include primary resection and anastomosis with diverting loop ileostomy and laparoscopic lavage or drainage as the primary intervention [4].

Parallel with these developments, rapid source control laparotomy (RSCL), an approach borrowed from trauma surgery for managing patients with catastrophic bleeding and devastating intra-abdominal injury [5], has been adapted for the management for acute diverticulitis. The so-called damage-control laparotomy (DCL) was first developed 80 years ago by Ogilvie [6], then reinvigorated by Stone et al. [7] and subsequently by Rotondo in 1993 [8]. Clinical evidence of 
a benefit using damage control approach in trauma patients with the lethal triad of hypothermia, metabolic acidosis, and coagulopathy was established in a cohort study. After initial operative management, patients were brought to an intensive care unit for aggressive resuscitation followed by definitive abdominal closure within $48 \mathrm{~h}$ if resuscitation was successful $[9,10]$.

The use of the DCL in patients with acute diverticulitis is an innovative approach which has emerged over the last 20 years with the emergence in the USA of acute care surgery services which provide care for all abdominal emergencies including patients with perforated diverticulitis [11-13]. Its use in patients who have inflamed peritoneum with exudate weeping from the surfaces, who are not hypothermic or coagulopathic is a significant shift in surgical management of acute diverticulitis. The purpose of this retrospective cohort analysis is to subject RSCL to the scrutiny of direct study.

\section{Methods}

\section{Patient selection criteria}

This retrospective cohort analysis was based on 3 years of data from 2014 through 2016, compiled from The American College of Surgeons National Surgical Quality Improvement Program (NSQIP). The data was used to assess patients who had colectomy for acute diverticulitis. Adult patients who had an open laparotomy with colon resection as an emergency procedure for perforated colon with fecal peritonitis, Hinchey 4 classification, were included in the study. All elective colon surgeries were excluded. The patient populations were sorted into two groups: PFC, patients who had their fascia closed primarily after their initial operation and RSCL, patients who had their fascia left open after the initial operation.

\section{Data analysis}

Patient demographic information and outcomes were summarized using summary statistics with median with interquartile range (first quartile (Q1)-third quartile (Q3)) for continuous variables, and frequency and percentage for categorical variables. To compare the groups, the Wilcoxon rank sum test was used for continuous variables, and the chi-square test was used for the categorical variables as described previously [11]. The normality of data was tested using the Shapiro-Wilk test. The propensity score for RSCL was calculated for each subject. Then the one-toone matching was performed using the "nearest neighbor" as the matching method to pair an RSCL subject with a PFC subject. Propensity matching was performed using patients' demography, comorbidities, transfusion requirements, ASA classification, and septic shock so that the matching the two groups on the same disease burden.

The propensity score matching was performed using the R package "MatchIt" [14]. The following variables were used for calculating the propensity score: gender, age, race, history of diabetes, history of smoking, history of ventilatory support, history of chronic obstructive pulmonary disease, history of ascites, history of congestive heart failure, history of hypertension requiring medication, history of renal failure, history of dialysis, disseminated cancer, steroid use, history of weight loss, bleeding disorder, history of transfusions, wound class, American Society of Anesthesia classification, and history of septic shock. After matching, the numeric and graphical diagnostics were used to evaluate the improvement. The patient demographic information and outcomes from the matched subjects were summarized as described above. The Wilcoxon signed rank test was used to compare the continuous variables between matched groups, depending on the normality of data. The McNemar test was used to compare the categorical variables between matched groups, if the level of a categorical variable is two. If the level of a categorical variable is more than two, the Stuart-Maxwell test was used. The risk difference and odds ratio with their respective $95 \%$ confidence intervals were calculated. For the length of total hospital stay, the Kaplan-Meier procedure was used to estimate the median time, and the standard error was estimated using the Greenwood formula. The Kaplan-Meier curves were generated. The log-rank test was used to compare the time (Kaplan-Meier curves) between groups. The 2 -sided $p$ value was reported for each test. A $p$ value of 0.05 or less was considered an indication of statistical significance. Statistical analysis was performed using the $R$ language [15].

\section{Results}

\section{Comparison of groups before matching}

The data was initially assessed without consideration of what operative procedures were performed. There was a significant difference in operative procedures performed in the PFC compared to the RSCL group. A much higher percentage of patients in the RSCL group had a primary anastomosis without a protective ostomy compared to the PFC group (33.8\% vs $7.1 \%$, respectively, $p<0.001$ ), and significantly fewer percentage of patients in the RSCL group had a resection with an end colostomy and a distal closure $(33.8 \%$ vs $68.8 \%$, respectively, $p<0.001$ ). In the RSCL patients $56.3 \%$ required ventilator support as compared to $20.4 \%$ in the $\mathrm{PFC}$ group. With this analysis the mortality rate was significantly higher in the RSCL group compared to the PFC (18.3\% vs 
$8.5 \%$, respectively, $p<0.024)$. The resultant morbidity was also higher due to an increased renal failure, $2.1 \%$ versus $9.2 \%, p=0.024$, and prolonged respiratory failure as determined by a requirement of respiratory support, $56.3 \%$ versus $20.4 \%, p<0.0001$.

Tables 1 and 2 show the data before and after group matching. Principle treatment modalities were evenly matched with a level of difference, $p<0.386$. There was no difference in the incidence of septic shock. The only significant difference in the groups before and after matching is in the origin of the patients. The PFC patients were more likely to come from home and not an outside emergency department. The mortality rates in the two groups were not different (Table 3).

\section{Length of stay and discharge destination}

There were significant differences in the discharge destination in the two groups. Most remarkable was the PFC patients were twice as likely to go home than the RSCL group. Other discharge differences are shown in Table 4. The postoperative length of stay was significantly longer $(p=0.02)$ in the RSCL group as compared to the PFC and data is shown as a Kaplan-Meier curve in Fig. 1. The total

Table 1 Comparison of groups before propensity matching

\begin{tabular}{|c|c|c|c|c|}
\hline Variable & Values & $\mathrm{PFC}(n=359)$ & $\operatorname{RSCL}(n=101)$ & $P$ value \\
\hline Age in years & Median [Q1-Q3] & $64[54-73]$ & $64[57-74]$ & 0.825 \\
\hline \multirow[t]{2}{*}{ Sex, $n(\%)$} & Female & $189(52.6)$ & $61(60.4)$ & 0.205 \\
\hline & Male & $170(47.4)$ & $40(39.6)$ & \\
\hline Race (White), $n(\%)$ & 1 & $309(86.1)$ & $89(88.1)$ & 0.714 \\
\hline \multirow[t]{5}{*}{ Origin of patient, $n(\%)$} & From acute care hospital inpatient & $36(10)$ & $11(10.9)$ & 0.108 \\
\hline & Not transferred (admitted from home) & $257(71.6)$ & $60(59.4)$ & \\
\hline & Nursing home-chronic care-intermediate care & $10(2.8)$ & $5(5)$ & \\
\hline & Outside emergency department & $50(13.9)$ & $22(21.8)$ & \\
\hline & Transfer from other & $6(1.7)$ & $3(3)$ & \\
\hline Septic shock, $n(\%)$ & Yes & $42(11.7)$ & 39 (38.6) & $<0.001$ \\
\hline Ventilator dependent prior to surgery & Yes & $8(2.2)$ & $7(6.9)$ & 0.027 \\
\hline \multicolumn{5}{|l|}{ Comorbidities, $n(\%)$} \\
\hline \multirow[t]{3}{*}{ Diabetes } & Insulin & $19(5.3)$ & $5(5)$ & 0.743 \\
\hline & No & $316(88)$ & $87(86.1)$ & \\
\hline & Non-insulin & $24(6.7)$ & $9(8.9)$ & \\
\hline Smoking & Yes & $76(21.2)$ & $23(22.8)$ & 0.834 \\
\hline COPD & Yes & $45(12.5)$ & $12(11.9)$ & 0.996 \\
\hline Ascites & Yes & $1(0.3)$ & $3(3)$ & 0.035 \\
\hline \multirow[t]{2}{*}{$\mathrm{CHF}$} & Yes & $8(2.2)$ & $5(5)$ & 0.171 \\
\hline & Yes & $8(2.2)$ & $5(5)$ & \\
\hline Hypertension requiring medication & Yes & $207(57.7)$ & $55(54.5)$ & 0.645 \\
\hline Renal failure & Yes & $12(3.3)$ & $5(5)$ & 0.549 \\
\hline Dialysis & Yes & $8(2.2)$ & $4(4)$ & 0.307 \\
\hline Disseminated cancer & Yes & $15(4.2)$ & $8(7.9)$ & 0.205 \\
\hline Steroid use & Yes & $59(16.4)$ & $22(21.8)$ & 0.272 \\
\hline Weight loss & Yes & 13 (3.6) & $4(4)$ & 0.773 \\
\hline Bleeding disorder & Yes & $47(13.1)$ & $19(18.8)$ & 0.198 \\
\hline Transfusion requirement, $n(\%)$ & Yes & $6(1.7)$ & $5(5)$ & 0.069 \\
\hline \multirow[t]{6}{*}{ ASA class, $n(\%)$} & 1-No disturb & $6(1.7)$ & $0(0)$ & 0.021 \\
\hline & 2-Mild disturb & $77(21.4)$ & $15(14.9)$ & \\
\hline & 3-Severe disturb & $166(46.2)$ & $37(36.6)$ & \\
\hline & 4-Life threat & $96(26.7)$ & 45 (44.6) & \\
\hline & 5-Moribund & $12(3.3)$ & $4(4)$ & \\
\hline & None assigned & $2(0.6)$ & $0(0)$ & \\
\hline
\end{tabular}

COPD Chronic obstructive pulmonary disease, CHF congestive heart failure, ASA class American Society of anesthesiologist classification, $Q 1-$ Q3 Interquartile range between 1st and 3rd quartile 
Table 2 Comparison of groups after propensity matching

\begin{tabular}{|c|c|c|c|c|}
\hline Variable & Values & $\operatorname{PFC}(n=359)$ & $\operatorname{RSCL}(n=101)$ & $P$ value \\
\hline Age in years & Median [Q1-Q3] & $64[55.8-73]$ & $64[57-73.3]$ & 0.818 \\
\hline \multirow[t]{2}{*}{ Sex, $n(\%)$} & Female & $50(54.3)$ & $56(60.9)$ & 0.470 \\
\hline & Male & $42(45.7)$ & $36(39.1)$ & \\
\hline Race (White), $n(\%)$ & Yes & $76(82.6)$ & $80(87)$ & 0.540 \\
\hline \multirow[t]{5}{*}{ Origin of patient, $n(\%)$} & From acute care hospital inpatient & $11(12)$ & $9(9.8)$ & $<0.001$ \\
\hline & Not transferred (admitted from home) & $58(63)$ & $54(58.7)$ & \\
\hline & Nursing home-chronic care-intermediate care & $4(4.3)$ & $4(4.3)$ & \\
\hline & Outside emergency department & $18(19.6)$ & $22(23.9)$ & \\
\hline & Transfer from other & $1(1.1)$ & $3(3.3)$ & \\
\hline Septic shock, $n(\%)$ & Yes & $31(33.7)$ & $31(33.7)$ & $>0.99$ \\
\hline Ventilator dependent prior to surgery & Yes & $6(6.5)$ & $5(5.4)$ & $>0.99$ \\
\hline \multicolumn{5}{|l|}{ Comorbidities, $n(\%)$} \\
\hline \multirow[t]{3}{*}{ Diabetes } & Insulin & $5(5.4)$ & $5(5.4)$ & 0.414 \\
\hline & No & $78(84.8)$ & $80(87)$ & \\
\hline & Non-insulin & $9(9.8)$ & $7(7.6)$ & \\
\hline Smoking & Yes & $17(18.5)$ & $20(21.7)$ & 0.677 \\
\hline COPD & Yes & $12(13)$ & $12(13)$ & $>0.99$ \\
\hline Ascites & Yes & $1(1.1)$ & $2(2.2)$ & $>0.99$ \\
\hline $\mathrm{CHF}$ & Yes & $6(6.5)$ & $3(3.3)$ & 0.505 \\
\hline Hypertension requiring medication & Yes & $52(56.5)$ & $51(55.4)$ & $>0.99$ \\
\hline Renal failure & Yes & $3(3.3)$ & $5(5.4)$ & 0.724 \\
\hline Dialysis & Yes & $2(2.2)$ & $3(3.3)$ & $>0.99$ \\
\hline Disseminated cancer & Yes & $7(7.6)$ & $8(8.7)$ & $>0.99$ \\
\hline Steroid use & Yes & $26(28.3)$ & $20(21.7)$ & 0.377 \\
\hline Weight loss & Yes & $5(5.4)$ & $3(3.3)$ & 0.724 \\
\hline Bleeding disorder & Yes & $13(14.1)$ & $15(16.3)$ & 0.838 \\
\hline Transfusion requirement, $n(\%)$ & Yes & $2(2.2)$ & $3(3.3)$ & $>0.99$ \\
\hline \multirow[t]{6}{*}{ ASA class, $n(\%)$} & 1-No disturb & $0(0)$ & $0(0)$ & 0.442 \\
\hline & 2-Mild disturb & $14(15.2)$ & $15(16.3)$ & \\
\hline & 3-Severe disturb & $39(42.4)$ & $35(38)$ & \\
\hline & 4-Life threat & $33(35.9)$ & $39(42.4)$ & \\
\hline & 5-Moribund & $6(6.5)$ & $3(3.3)$ & \\
\hline & None assigned & $0(0)$ & $0(0)$ & \\
\hline
\end{tabular}

$C O P D$ Chronic obstructive pulmonary disease, $C H F$ congestive heart failure, ASA class American Society of anesthesiologist classification, $Q 1-$ Q3 interquartile range between 1 st and 3rd quartile

Table 3 30-day mortality and length of stay

\begin{tabular}{lllll}
\hline Variable & Values & PFC $(n=92)$ & RSCL $(n=92)$ & P value \\
\hline Died, $n(\%)$ & No & $83(90.2)$ & $77(83.7)$ & 0.286 \\
& Yes & $9(9.8)$ & $15(16.3)$ & \\
Days from operation to death & Median (95\% CI) & $8[6, \mathrm{NA}]$ & $7[3, \mathrm{NA}]$ & 0.300 \\
& $\begin{array}{l}\text { [Kaplan-Meier } \\
\text { procedure }]\end{array}$ & & \\
Total length of hospital stay & $\begin{array}{c}\text { Median (95\% CI) } \\
\text { [Kaplan-Meier }\end{array}$ & $13[8,12]$ & & 0.020 \\
& procedure] & & \\
\hline
\end{tabular}

$N A$ not available 
Table 4 Discharge destination of patients who survived

\begin{tabular}{lllll}
\hline Variable & Value & PFC $(n=83)$ & RSCL $(n=77)$ & P value \\
\hline Discharge destination & Facility which was home & $1(1.2)$ & $1(1.3)$ & 0.002 \\
& Home & $40(48.2)$ & $19(24.7)$ & \\
& Hospice & $1(1.2)$ & $1(1.3)$ & \\
& Rehab & $6(7.2)$ & $19(24.7)$ & \\
& Separate acute care & $2(2.4)$ & $4(5.2)$ & \\
& Skilled care, not home & $33(39.8)$ & $32(41.6)$ & \\
& Unskilled facility not home & $0(0)$ & $1(1.3)$ & \\
& Value & PFC (n=92) & RSCL (n=92) & $P$ value \\
Variable & Median (95\% CI) [Kaplan- & $12[8,12]$ & $15[10,16]$ & 0.020 \\
Days postoperative to discharge & & & \\
& Meier procedure] & &
\end{tabular}

Fig. 1 Hospital length of stay between the groups

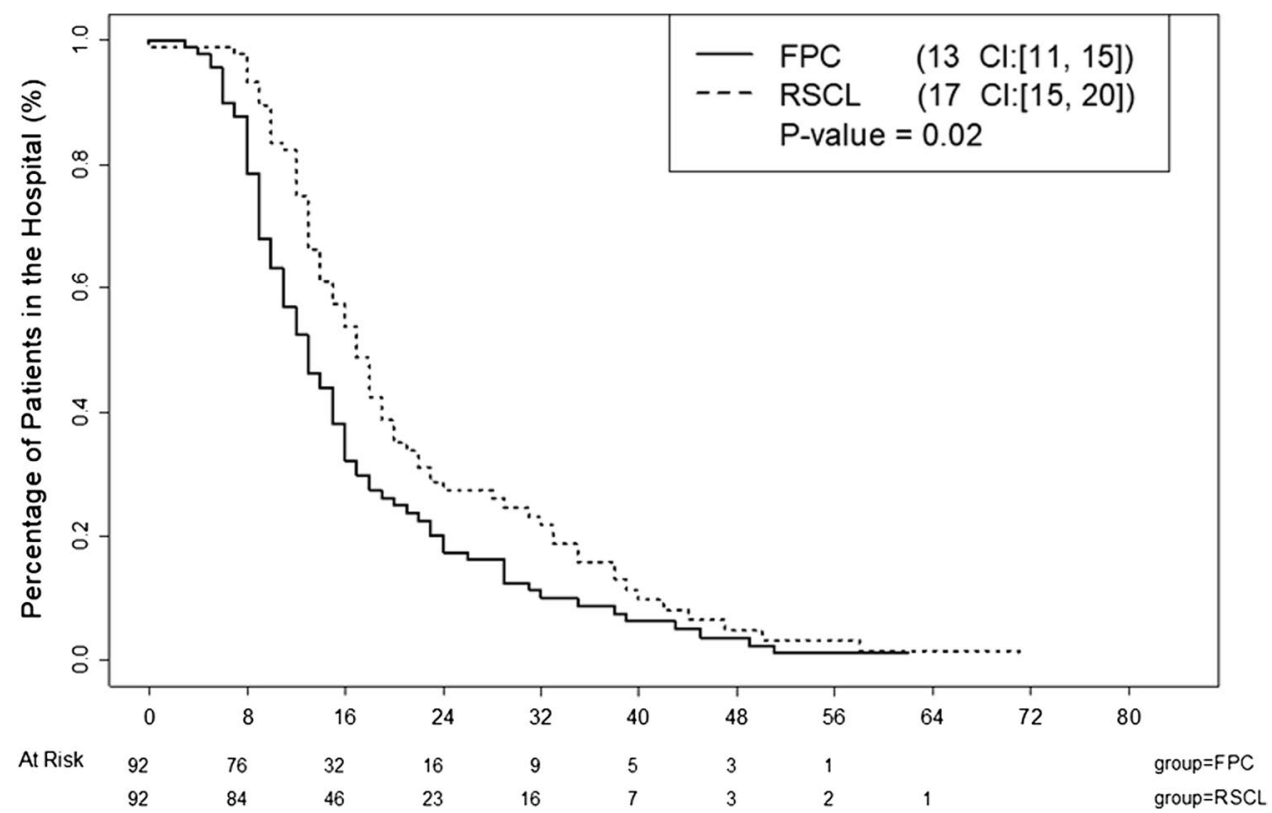

length of stay, displayed in Fig. 1, was shorter for PFC versus RSCL patients (13 versus 17 days, $p=0.02$ ).

\section{Discussion}

There has been a growing interest in the role of RSCL in treating acute perforated diverticulitis as evidenced by the number of recent publications exploring the efficacy of this approach [16-24] Many of these studies are systematic reviews or meta-analyses and consequently designed to assess consensus opinion. In three studies [16-18], RSCL was deemed a safe approach which resulted in more patients being "stoma-free" after surgery than the traditional approach. One cohort study compared two different negative pressure devices for the treatment of perforated diverticulitis. No differences were identified between the devices, but no analysis was made with respect to open versus closed abdomen [22]. In another study, the presence of ongoing peritonitis at the time of the second surgery for abdominal wall closure was associated with a significantly higher rate of morbidity and mortality. The complication rate was nearly doubled in this group. The authors did find that without ongoing peritonitis an anastomosis could be safely performed [23]. Most recently, the use of a vacuum device for temporary coverage was assessed in a large systemic review. Zizzo et al. found RSCL to be effective but may have been used too frequently in a significant number of patients who did not necessarily need it [24].

A general consensus from these studies suggests that while there is merit in the use of temporary closure for Hinchey III and IV diverticulitis, universal acceptance has not been achieved. Our study describes some of the negative consequences of RSCL which are important to consider when devising a treatment algorithm for patients with severe diverticulitis. The downside of RSCL is a prolonged hospital stay and deconditioning of the patient. These disadvantages must be weighed against the severity of the patients' disease. 
Our observations were consistent with prior publications where the use of open abdomen in a broad range of nontraumatic acute surgical situations was evaluated [25-28]. Restricting RSCL to patients who meet the trauma criteria of acidosis, coagulopathy and hypotension for damage control surgery may significantly reduce inappropriate use of this valuable tool [5-8].

An unexpected finding in our analyses was that a significant number of patients had a primary anastomoses performed without a proximal stoma. This was a concerning approach since all of these patients had feculent peritonitis. The question of whether an "unprotected" anastomosis should be done is controversial [29]. In the context of patients with Hinchey III diverticulitis data supports primary anastomosis without RSCL [19]. However, these patients had a laparoscopic lavage and drainage as a separate procedure prior to their surgical resection and anastomosis. While there is evidence to support placing a primary unprotected anastomosis in traumatic colon injuries [30], this approach may not translate to patients with acute perforated diverticulitis. Patients in the Anjaria study were half the age of the patients reported here and did not have an active infectious process causing the bowel perforation. Our data suggests that the open abdomen is not at fault. Rather, the unreal expectations that a primary anastomosis in an elderly patient will hold together simply by leaving the abdomen open was the underlying failure in clinical management and should not be done in this setting. After our data was analyzed by controlling for the type of operative procedure performed, there was no difference in mortality and the major differences in morbidity were discharge destination, further suggesting an unprotected anastomosis put the patient at risk for septic complications.

The inherent limitations are related to its retrospective of a database. This limits the data to in-hospital patients and is subject to coding bias as well as limited long-term follow-up (for NSQIP, it is 30 days). Also, propensity analysis has its own flaws with risk adjustment. Despite having 20 different demographic and clinical variables, it is impossible to measure all confounders which would result in a comparison of two dissimilar groups. Furthermore, our patient population was only moderately sized, and there was also variation in time to fascial closure as well as different management styles of open abdomen that could not be accounted for.

This study has identified that over $20 \%$ of patients admitted with acute diverticulitis are treated with RSCL. Patients treated with RSCL had a longer hospital stays. More patients treated with RSCL were more likely to be discharged to a rehabilitation facility than PFC patients. The use of RSCL at a rate of $20 \%$ is very high. Its use should be limited to critically ill patients too unstable to undergo anything more than source control or are at risk for abdominal compartment syndrome.

\section{Declarations}

Ethical approval All procedures performed in studies involving human participants were in accordance with the ethical standards of the institutional and/or national research committee and with the 1964 Helsinki declaration and its later amendments or comparable ethical standards.

Conflict of interest The authors declare no competing interests.

\section{References}

1. Masoomi H, Buchberg B, Magno C, Mills S, Stamos M (2011) Trends in diverticuliits management in the United States from 2002 to2007. JAMA 146(4):400-406+

2. Etzioni DA, MacK TM, Beart RW, Kaiser AM (2009) Diverticulitis in the United States: 1998-2005: changing patterns of disease and treatment. Ann Surg 249(2):210-217

3. Cirocchi R, Afshar S, Di Saverio S, Popivanov G, De Sol A, Gubbiotti $\mathrm{F}$ et al (2017) A historical review of surgery for peritonitis secondary to acute colonic diverticulitis: from Lockhart-Mummery to evidence-based medicine. World J Emerg Surg 12:14

4. Oberkofler CE, Rickenbacher A, Raptis DA, Lehmann K, Villiger $\mathrm{P}$, Buchli $\mathrm{C}$ et al (2012) A multicenter randomized clinical trial of primary anastomosis or Hartmann's procedure for perforated left colonic diverticulitis with purulent or fecal peritonitis. Ann Surg (5):819-826

5. Becher RD, Peitzman AB, Sperry JL, Gallaher JR, Neff LP, Sun $Y$ et al (2016) Damage control operations in non-trauma patients: defining criteria for the staged rapid source control laparotomy in emergency general surgery. World J Emerg Surg 11:10-18

6. Ogilvie WH (1940) The late complications of abdominal warwounds. Lancet 236:253-257

7. Stone HH, Strom PR, Mullins RJ (1983) Management of the major coagulopathy with onset during laparotomy. Ann Surg 197(5):532-535

8. Rotondo MF, Schwab CW, McGonigal MD, Phillips GR, Fruchterman TM, Kauder DR et al (1993) 'Damage control': an approach for improved survival in exsanguinating penetrating abdominal injury. J Trauma Injury Infect Crit Care 35(3):375-382

9. Godat L, Kobayashi L, Costantini T, Coimbra R (2013) Abdominal damage control surgery and reconstruction: world society of emergency surgery position paper. World J Emerg Surg 8(1):53

10. Cipolla J, Stawicki SP, Hoff WS, McQuay N, Hoey BA, Wainwright $\mathrm{G}$ et al (2005) A proposed algorithm for managing the open abdomen. American Surgeon 71(3):202-209

11. Institute of Medicine (2006) IOM Report: The future of emergency care in the United States health system. Academic Emergency Medicine 48(2):115-120

12. Khubchandani JA, Ingraham AM, Daniel VT, Ayturk D, Kiefe CI, Santry HP (2018) Geographic diffusion and implementation of acute care surgery: an uneven solution to the national emergency general surgery crisis. JAMA Surg 153(2):150-159

13. Ball CG, Hameed SM, Brenneman FD (2010) Acute care surgery: a new strategy for the general surgery patients left behind. Can J Surg 25(2):84-85

14. Ho DE, Imai K, King G, Stuart EA (2011) MatchIt: Nonparametric preprocessing for parametric causal inference. J Stat Softw 42(8):1-28 
15. R Development Core Team R (2011) R: A language and environment for statistical computing. R Foundation for Statistical Computing

16. Sohn M, Agha A, Iesalnieks I, Gundling F, Presl J, Hochrein A et al (2021) Damage control strategy in perforated diverticulitis with generalized peritonitis. BMC Surg [cited 2021 Jun 16]; 21(1):1-11. Available from: https://doi.org/10.1186/ s12893-021-01130-5

17. Gachabayov M, Oberkofler CE, Tuech JJ, Hahnloser D, Bergamaschi R (2018) Resection with primary anastomosis vs nonrestorative resection for perforated diverticulitis with peritonitis: a systematic review and meta-analysis. Vol. 20, Colorectal Disease. Blackwell Publishing Ltd; [cited 2021 Jun 16]. p. 753-70. Available from: https://onlinelibrary.wiley.com/doi/full/10.1111/codi. 14237

18. Cirocchi R, Arezzo A, Vettoretto N, Cavaliere D, Farinella E, Renzi C et al (2014) Role of damage control surgery in the treatment of Hinchey III and IV sigmoid diverticulitis: a tailored strategy. Medicine (United States) 93(25):e184

19. Acuna SA, Wood T, Chesney TR, Dossa F, Wexner SD, Quereshy FA et al (2018) Operative strategies for perforated diverticulitis: a systematic review and meta-analysis. Dis Colon Rectum. [cited 2021 Jun 16];61(12):1442-53. Available from: https://journals. lww.com/dcrjournal/Fulltext/2018/12000/Operative_Strategies_ for_Perforated.17.aspx

20. Coccolini F, Roberts D, Ansaloni L, Ivatury R, Gamberini E, Kluger $Y$ et al (2018) The open abdomen in trauma and nontrauma patients: WSES guidelines. Vol. 13, World Journal of Emergency Surgery. BioMed Central Ltd.; [cited 2021 Jun 16]. p. 1-16. Available from: https://link.springer.com/articles/10.1186/ s13017-018-0167-4

21. Kafka-Ritsch R, Birkfellner F, Perathoner A, Raab H, Nehoda H, Pratschke J et al (2012) Damage control surgery with abdominal vacuum and delayed bowel reconstruction in patients with perforated diverticulitis Hinchey III/IV. J Gastrointest Surg [cited 2021 Jun 16];16(10):1915-22. Available from: https://link.sprin ger.com/article/10.1007/s11605-012-1977-4

22. Gasser E, Alexander P, Reich-Weinberger S, Buchner S, Kogler $\mathrm{P}$, Zitt M et al (2019) Damage control surgery for perforated diverticulitis: a two center experience with two different abdominal negative pressure therapy devices. Acta Chirurgica Belgica [cited 2021 Jun 16];119(6):370-5. Available from: https://www. tandfonline.com/action/journalInformation?journalCode $=$ tacb20

23. Sohn MA, Agha A, Steiner P, Hochrein A, Komm M, Ruppert R et al (2018) Damage control surgery in perforated diverticulitis: ongoing peritonitis at second surgery predicts a worse outcome. Int J Colorectal Dis 33(7):871-878

24. Zizzo M, Castro Ruiz C, Zanelli M, Bassi MC, Sanguedolce F, Ascani $S$ et al (2020) Damage control surgery for the treatment of perforated acute colonic diverticulitis: a systematic review. Medicine 99(48): 23323

25. Vogler J, Bagwell L, Hart L, Holmes S, Sciarretta J, Davis J (2017) Rapid Source-control laparotomy: is there a mortality benefit? Surg Infect (7):787-92

26. Vogler J, Hart L, Holmes S, Sciaretta J, Davis JM (2018) Rapid source-control laparotomy: is there a mortality benefit in septic shock? Surg Infect 19(2):225-229

27. Kao AM, Cetrulo LN, Baimas-George MR, Prasad T, Heniford BT, Davis BR et al (2019) Outcomes of open abdomen versus primary closure following emergent laparotomy for suspected secondary peritonitis. J Trauma Acute Care Surg 87(3):623-629

28. Robledo FA, Luque-de-León E, Suárez R, Sánchez P, de-la-Fuente M, Vargas A et al (2007) Open versus closed management of the abdomen in the surgical treatment of severe secondary peritonitis: a randomized clinical trial. Surg Infect 35(3):375-382

29. Binda GA, Karas JR, Serventi A, Sokmen S, Amato A, Hydo L et al (2012) Primary anastomosis vs nonrestorative resection for perforated diverticulitis with peritonitis: a prematurely terminated randomized controlled trial. Colorectal Dis 14(11):1403-1407

30. Anjaria D, Ullmann T, Lavery R et al (2014) Management of colonic injuries in the settng of damage-control laparotomyOne shot to get it right. J Trauma Acute Care Surg 76(3):594-600

Publisher's note Springer Nature remains neutral with regard to jurisdictional claims in published maps and institutional affiliations. 